\title{
Management of a microprolactinoma with aripiprazole in a woman with cabergoline-induced mania
}

\section{Lisa Burback}

Alberta Hospital Edmonton, Addiction and Mental Health Program, Alberta Health Services, 17480 Fort Road Post Office Box 307, Edmonton, Alberta, Canada T5J 2J7
Correspondence

should be addressed

to L Burback

Email

lisa.burback@

albertahealthservices.ca

\section{Summary}

A previously healthy 32-year-old woman developed cyclical mood swings after being prescribed cabergoline for a pituitary microprolactinoma. These mood swings persisted for over 2 years, at which point she developed an acute manic episode with psychotic features and was admitted to a psychiatry unit. Cabergoline was discontinued and replaced with aripiprazole $10 \mathrm{mg} /$ day. Her manic episode quickly resolved, and she was discharged within 6 days of admission. The aripiprazole suppressed her prolactin levels for over 18 months of follow-up, even after the dose was lowered to $2 \mathrm{mg} / \mathrm{day}$. There was no significant change in tumor size over 15 months and treatment was well tolerated. However, after 9 months of taking $2 \mathrm{mg}$ aripiprazole, she developed brief manic symptoms, and the dose was returned to $10 \mathrm{mg}$ daily, with good effect.

\section{Learning points:}

- Dopamine agonists such as cabergoline, which are a standard treatment for microprolactinomas, can have serious adverse effects such as psychosis or valvular heart disease.

- Aripiprazole is a well-tolerated atypical antipsychotic that, unlike other antipsychotics, is a partial dopamine agonist capable of suppressing prolactin levels.

- Adjunctive, low-dose aripiprazole has been utilized to reverse risperidone-induced hyperprolactinemia.

- This case report demonstrates how aripiprazole monotherapy, in doses ranging from 2 to $10 \mathrm{mg} /$ day, was effective in suppressing prolactin in a woman with a microprolactinoma who developed psychiatric side effects from cabergoline.

\section{Background}

Microprolactinomas are often treated with dopamine agonists, which can have serious medical and psychiatric adverse effects, including psychosis, impulse control disorders, dyskinesia, pulmonary fibrosis and valvular heart disease. Aripiprazole, a well-tolerated partial dopamine agonist, is an atypical antipsychotic that can reduce prolactin levels. Even small doses have been used adjunctively to treat antipsychotic-induced hyperprolactinemia. However, aripiprazole has not been evaluated for the general treatment of hyperprolactinemia in patients with microprolactinomas. Nor has it been studied for the treatment of hyperprolactinemia in patients without psychiatric illness. This case report illustrates its successful use in suppressing prolactin in a patient with no previous psychiatric history who developed severe psychiatric side effects from cabergoline. 
Aripiprazole may be a novel and well tolerated therapy for the treatment of hyperprolactinemia due to microprolactinomas. Further research is warranted.

\section{Case presentation}

A 32-year-old, otherwise healthy woman with no previous psychiatric history developed mania after treatment with cabergoline for a microprolactinoma. She was a professional woman, and was married with two young children. She had a remote history of migraine, was a nonsmoker and had no history of substance abuse. The family history was positive for depression, possible bipolar disorder and alcohol dependence.

At the time of her diagnosis, she reported a 1-year history of frontal headaches and galactorrhea, and 2 years of amenorrhea, starting after the birth of her second child. Her first prolactin level was $48.1 \mu \mathrm{g} / \mathrm{l}$ (reference $<25 \mu \mathrm{g} / \mathrm{l}$ ) at the time of referral to endocrinology, $\sim 7$ months before her diagnosis. (See Table 1 for the results of endocrine laboratory investigations.) A pituitary MRI revealed an ' $8 \times 4 \mathrm{~mm}$ heterogeneous lesion with a fluid level' suggestive of a microprolactinoma with subacute hemorrhage (Figs 1 and 2).

She was prescribed cabergoline $0.25 \mathrm{mg}$ orally per week. Two months later, her prolactin level was $26.3 \mu \mathrm{g} / \mathrm{l}$ (reference $<25 \mu \mathrm{g} / \mathrm{l}$ ) so the dose was increased to $0.25 \mathrm{mg}$ twice weekly. At her 6-month appointment, she complained of 'mood changes,' especially depressed mood in the 2 weeks prior to her menses. Therefore, cabergoline was reduced to $0.25 \mathrm{mg}$ weekly. Her prolactin levels remained suppressed, and repeat MRIs 6 and 18 months after diagnosis showed no change.

However, she continued to have cyclical mood fluctuations. She would experience 2 weeks of normal mood, followed by a week of depressed mood with hypersomnia, anorexia and fatigue, and then a week of hypomanic symptoms. During her hypomanic phase, she reported a decreased need for sleep, increased energy, racing thoughts, distractibility, over-talkativeness and feeling like she was 'strong and capable.'

In the week before hospitalization, she rapidly developed mania with pressured speech, agitation, labile affect, a decreased need for sleep and grandiose, religious and persecutory delusions. She described seeing Jesus in a white shadow and hearing ringing sounds that she believed were an announcement of His coming. She believed she could predict the future and talk to Jesus. She thought her husband was 'the Antichrist' who might
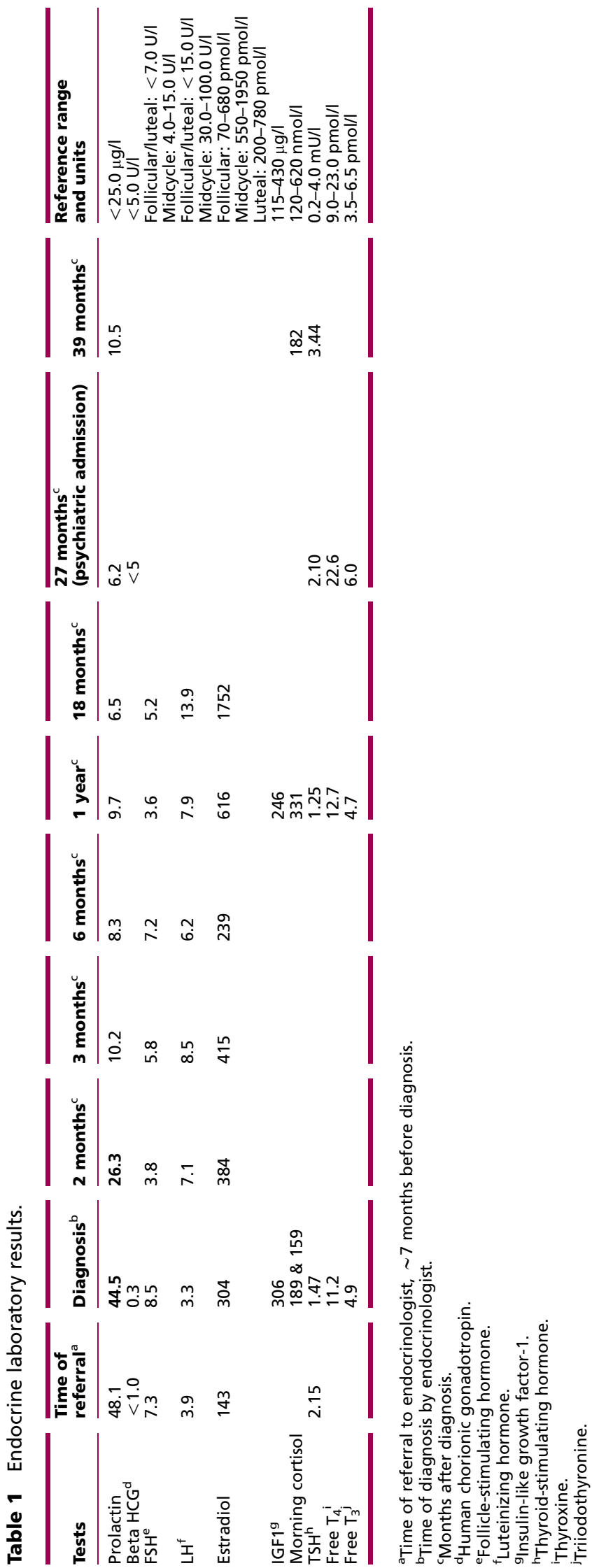


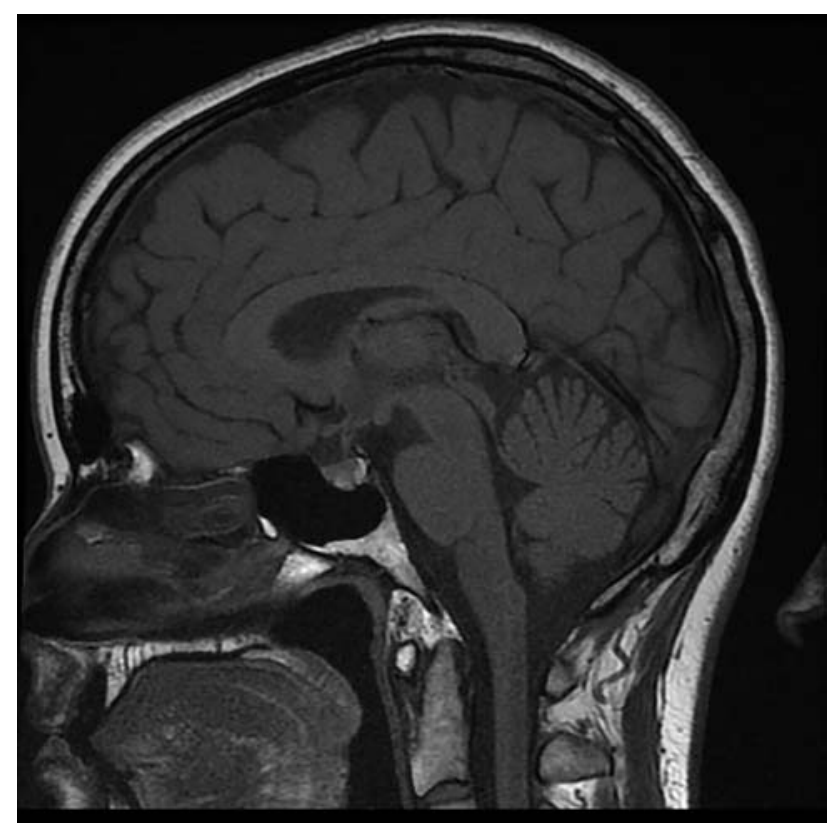

Figure 1

Sagital MRI image of pituitary microprolactinoma.

harm their two children. She was admitted to psychiatry, and cabergoline was discontinued.

\section{Investigation}

Laboratory investigations at the time of admission revealed a prolactin level of $6.2 \mu \mathrm{g} / \mathrm{l}$ (reference $<25 \mu \mathrm{g} / \mathrm{l}$ ). See Table 1 for results of other endocrine laboratory investigations. In addition, calcium was $2.44 \mathrm{mmol} / \mathrm{l}$ (reference $2.10-2.60 \mathrm{mmol} / \mathrm{l}$ ), random glucose $5.4 \mathrm{mmol} / \mathrm{l}$ (reference $3.3-11.0 \mathrm{mmol} / \mathrm{l}$ ), sodium $140 \mathrm{mmol} / \mathrm{l}$ (reference $133-146 \mathrm{mmol} / \mathrm{l}$ ), potassium $4.0 \mathrm{mmol} / \mathrm{l}$ (reference 3.3-4.8 mmol/l), creatinine $68 \mu \mathrm{mol} / \mathrm{l}$ (reference 50-105 umol/l), vitamin B12 640 pmol/l (reference $>150 \mathrm{pmol} / \mathrm{l}$ ), ferritin $66 \mu \mathrm{g} / \mathrm{l}$ (reference $12-300 \mu \mathrm{g} / \mathrm{l}$ ) and folate was $>40 \mathrm{nmol} / 1$ (reference $<10 \mathrm{nmol} / 1$ for deficiency). C-reactive protein was undetectable, and a complete blood count, urinalysis and a urine drug screen were unremarkable.

\section{Treatment}

In the emergency department, the patient briefly received oral clonazepam and olanzapine to reduce agitation. However, this was quickly switched to aripiprazole $10 \mathrm{mg}$ orally per day as monotherapy. The rationale was that aripiprazole is a partial dopamine agonist that can suppress prolactin. Other antipsychotic treatments block dopamine receptors and can cause hyperprolactinemia to varying degrees. It was hoped that aripiprazole would treat her manic symptoms while also suppressing her prolactin levels, avoiding the need for a full dopamine agonist such as cabergoline. The manic symptoms quickly resolved, and she was discharged home 6 days after admission. Given the rapid improvement in her symptoms after cabergoline was discontinued, she was diagnosed with a cabergolineinduced bipolar disorder.

\section{Outcome and follow-up}

After discharge from hospital, she was instructed to keep a mood diary that included mood, neurovegetative function and her menstrual cycle. During the next few months, there was no evidence of premenstrual dysphoric disorder, clinical depression or hypomania. However, she did report generalized anxiety in reaction to her manic episode. She was treated with escitalopram $10 \mathrm{mg}$ orally daily, with excellent effect.

Follow-up investigations related to her microprolactinoma revealed stability. Prolactin was $11.4 \mu \mathrm{g} / \mathrm{l}$ (reference $<25 \mu \mathrm{g} / \mathrm{l}) 2$ months after discharge. A follow up MRI 3 months post discharge, which was 2.5 years after the microprolactinoma diagnosis, showed a slight reduction

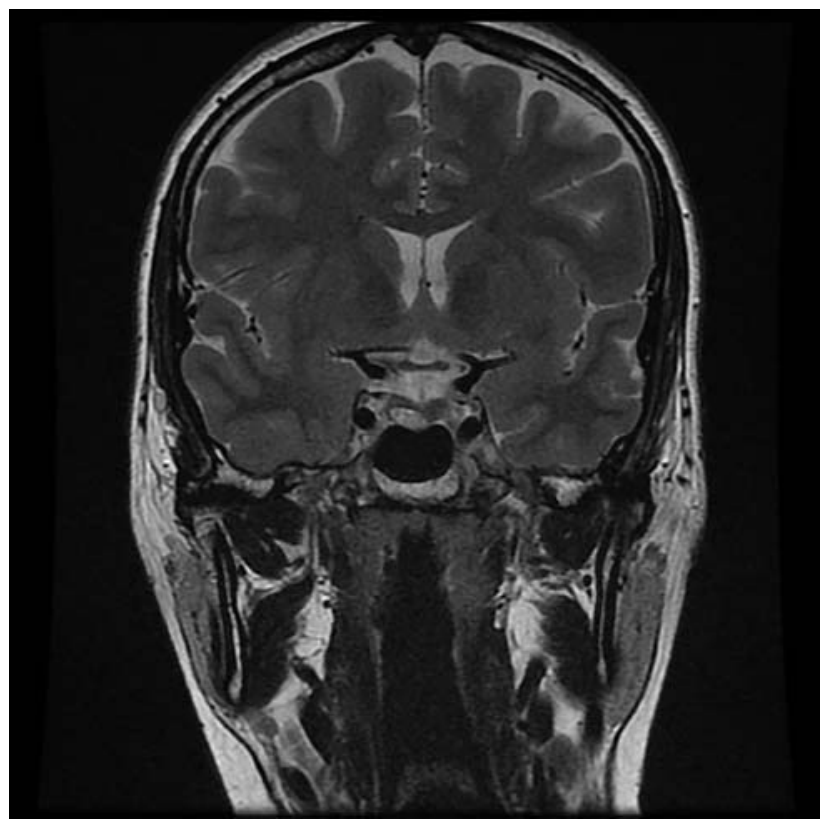

Figure 2

Coronal T2 MRI image of patient's microprolactinoma. 
in tumor size to ' $6 \mathrm{~mm}$ in maximal dimension,' which was 'more heterogeneous and less conspicuous when compared to the previous study.'

After a few months of escitalopram treatment, she felt emotionally blunted and fatigued. Therefore, escitalopram was weaned and discontinued, without sequelae. She continued aripiprazole $10 \mathrm{mg}$ daily, which was eventually weaned to a final dose of $2 \mathrm{mg} /$ day. Laboratory investigations at 1 year post-discharge revealed a prolactin level of $10.5 \mu \mathrm{g} / \mathrm{l}$ (reference $<25 \mu \mathrm{g} / \mathrm{l}$ ), with normal TSH and cortisol (Table 1). A follow-up MRI 15 months after her discharge reported a $6 \mathrm{~mm}$ pituitary lesion, which was considered essentially unchanged. She remained medically and psychiatrically stable, with no further signs or symptoms of hyperprolactinemia, for 18 months of follow-up after her hospitalization. Then suddenly after 9 months of taking aripiprazole $2 \mathrm{mg}$ daily, she developed 4 days of manic symptoms, and her dose was increased to $10 \mathrm{mg}$ per day, which restored euthymia.

\section{Discussion}

Microprolactinomas are often treated with ergot-derived dopamine agonists such as cabergoline or bromocriptine. These drugs activate D2 receptors in the pituitary gland, which inhibits adenyl cyclase activity and results in reduced gene transcription and prolactin secretion. However, dopamine agonists also affect other dopamine, serotonin and adrenergic receptors, which can lead to adverse effects. Stimulation of D1 receptors can cause postural hypotension or dyskinesia $(1,2)$. Mesocortical and mesolimbic D2 and D3 receptors are thought to be involved in mood and behavior, and dopamine agonists can trigger psychosis or mania $(1,2,3)$. Impulse control disorders (ICDs), including problem gambling, hypersexuality, compulsive eating and compulsive shopping, may be mediated by D3 receptors in the mesolimbic system $(2,3)$. However, problem gambling has also been linked to D2 and D4 receptors (2). Noronha published a relevant review, which includes cases of ICDs that developed during treatment with dopamine agonists prescribed for prolactinomas (3). D4 stimulation has also been associated with 'sudden onset sleep' or 'sleep attacks' (2).

Ergot-derived dopamine agonists also influence various serotonin receptor subtypes. Retroperitoneal and pulmonary fibrosis and valvular heart disease may be related to stimulatory effects on $5-\mathrm{HT}_{1 \mathrm{~B}}$ and $5-\mathrm{HT}_{2 \mathrm{~B}}$ receptors. Valvulopathy is thought to be partly due to $5-\mathrm{HT}_{2 \mathrm{~B}}$-induced mitogenesis in cardiac cells, leading to an overgrowth of tissue (2). Dopamine agonists may also cause hallucinations via stimulatory effects on $5 \mathrm{HT}_{2 \mathrm{~A}}$ and $5 \mathrm{HT}_{2 \mathrm{C}}$ receptors. Kvernmo published a review of dopamine agonist receptor binding properties, which details the differences between them (2). In contrast aripiprazole blocks $5 \mathrm{HT}_{2 \mathrm{~A}}$ receptors.

Patients who develop mania or psychosis as a consequence of dopamine agonists receive the diagnosis of a substance-induced mood disorder or substance-induced psychosis. However, it is possible that some of these patients have an underlying psychiatric illness that was triggered by the dopamine agonist. In this case, the patient did not have a psychiatric history prior to cabergoline therapy. However, there was a family history of bipolar disorder. Regardless, the manic episode resolved very quickly after cabergoline was discontinued, giving support to the initial diagnosis of cabergoline-induced bipolar disorder.

However, the patient developed four days of manic symptoms nine months after her aripiprazole dose was reduced to $2 \mathrm{mg} /$ day. There have been case reports of aripiprazole causing mania in susceptible people, possibly due to partial dopamine agonism in combination with 5- $\mathrm{HT}_{1 \mathrm{~A}}$ agonism and 5- $\mathrm{HT}_{2 \mathrm{~A}}$ antagonism (4). However, this is rare, and her family history points to an underlying bipolar disorder that was triggered by cabergoline. The dose was increased to $10 \mathrm{mg} / \mathrm{day}$, which is considered a mood-stabilizing dose.

Psychosis usually requires antipsychotic medications, most of which raise prolactin levels and possibly promote tumor growth (1). It is theorized that antipsychotics raise prolactin by blocking dopamine, which ordinarily suppresses prolactin levels. A comprehensive review of the impact of various antipsychotics on prolactin can be found elsewhere (5). In contrast to most antipsychotics, aripiprazole is a partial dopamine agonist, especially at low doses. It has been shown to reduce prolactin levels in patients with and without hyperprolactinemia $(5,6)$.

Several studies have examined the use of aripiprazole augmentation in patients with risperidone-induced hyperprolactinemia $(7,8,9,10)$. In one study, 16 female patients with schizophrenia and risperidone-induced hyperprolactinemia received aripiprazole, which was increased from 3 to $12 \mathrm{mg} /$ day, in $3 \mathrm{mg}$ increments over 2 - to 4 -week intervals. Aripiprazole reduced prolactin levels at all four doses trialed, with a plateau effect at $6 \mathrm{mg} /$ day (7). This is in keeping with the theory that aripiprazole exhibits more dopamine agonism at lower doses than at higher doses.

Li et al. conducted a meta-analysis of five randomized controlled trials investigating the safety and efficacy of aripiprazole for antipsychotic induced hyperprolactinemia. Trials utilized aripiprazole in doses ranging from 2 
to $30 \mathrm{mg} /$ day. Aripiprazole was superior to placebo with respect to prolactin normalization (risk difference 0.76; 95\% CI $0.67-0.85 ; P<0.00001)$. A secondary analysis of patients receiving only $5 \mathrm{mg} /$ day was similar (risk difference $0.74 ; 95 \%$ CI $0.62-0.87 ; P<0.00001)$. There was no overall difference in adverse events or discontinuation rates between aripiprazole and placebo groups. Sedation, insomnia and headache were more frequent for doses $>15 \mathrm{mg} /$ day. The authors suggested that lowdose aripiprazole $5 \mathrm{mg}$ /day may be optimal (8).

However, this is controversial. A randomized, double blind trial of 119 schizophrenia patients with risperidone-induced hyperprolactinemia investigated the dose response relationship between aripiprazole and prolactin levels. Risperidone-treated patients were randomized to either placebo or aripiprazole 5,10 or $20 \mathrm{mg} /$ day for 8 weeks. All three aripiprazole doses reduced prolactin within 2 weeks. In contrast to the previous studies, the effect on prolactin was greater in the $10 \mathrm{mg}$ - and $20 \mathrm{mg} /$ day groups, relative to the $5 \mathrm{mg} /$ day group (9). In a case series, Lozano reported that an average dose of $17 \mathrm{mg} /$ day was effective in reducing risperidoneinduced hyperprolactinemia (10).

Reports also raise the possibility of prolactin deficiency in aripiprazole-treated patients (10). Lozano suggested, based on a study of 161 outpatients taking various atypical antipsychotics with or without aripiprazole, that the odds of a prolactin level of $<3 \mu \mathrm{g} / \mathrm{l}$ was 0.81 in patients receiving aripiprazole $(n=20)$ and 0.029 for those without aripiprazole $(n=141)$. Authors concluded that patients receiving at least $17 \mathrm{mg}$ daily had a high likelihood of prolactin deficiency (odds ratio, 28; 95\% CI, 7.42-105.78; $P<0.001)$, which could cause infertility, menstrual disorders, decreased testosterone and oligospermia (10). Interestingly, there are also reports of aripiprazole causing hyperprolactinemia, especially at higher doses $(1,5,11)$.

Aripiprazole has been used to treat psychosis in patients with established primary psychotic illnesses and comorbid microprolactinomas, often in combination with a dopamine agonist $(1,6)$. Chen et al. also reported on a woman with established bipolar disorder and comorbid microprolactinoma who developed mania and was treated with aripiprazole monotherapy. In her case, when the dose was lowered from 30 to $5 \mathrm{mg} /$ day, prolactin levels fell more dramatically and the hyperprolactinemia symptoms abated (12). This supports the idea that lower doses are more prodopaminergic.

Some authors have suggested that aripiprazole may be the treatment of choice for patients with comorbid microprolactinoma and psychosis $(5,6)$. However, adding or switching to aripiprazole may cause worsening of psychotic symptoms, agitation or activation in patients with established schizophrenia or schizoaffective disorder. In a systematic review of reported cases, a higher risk was associated with severe or treatment-resistant psychotic illness, higher doses of antipsychotics, use of conventional neuroleptics, and longer illness course. This may be due to aripiprazole's weaker antipsychotic effect, partial dopamine agonism, or the higher affinity for the D2 receptor. Alternatively, it could be related to dopamine upregulation in the context of long-term, high-potency neuroleptic use in severely ill patients (13).

To this author's knowledge, this is the first reported case of aripiprazole monotherapy for the treatment of microprolactinoma in a patient without a prior psychiatric history. In her case, cabergoline caused mood cycling and eventually a severe manic episode. Aripiprazole, in doses ranging from 2 to $10 \mathrm{mg}$ daily, successfully suppressed her prolactin levels over 18 months, and tumor size remained stable over 15 months of follow-up. Given the serious side effects of dopamine agonists, the relative safety of low-dose aripiprazole, and the short list of treatment options, it should be investigated as a general treatment option for hyperprolactinemia due to microprolactinoma.

Declaration of interest

The author declares that there is no conflict of interest that could be perceived as prejudicing the impartiality of the research reported.

\section{Funding}

This research did not receive any specific grant from any funding agency in the public, commercial or not-for-profit sector.

\section{Patient consent}

Consent was obtained from the patient described.

\section{References}

1 Ali S, Klahr K \& Freudenreich O 2010 Management of psychosis associated with a prolactinoma: case and review of the literature. Psychosomatics 51 370-376. (doi:10.1016/S0033-3182(10)70718-0)

2 Kvernmo T, Houben J \& Sylte I 2008 Receptor-binding and pharmacokinetic properties of dopaminergic agonists. Current Topics in Medicinal Chemistry 8 1049-1067. (doi:10.2174/156802608785161457)

3 Noronha S, Stokes V, Karavitaki N \& Grossman A 2015 Treating prolactinomas with dopamine agonists: always worth the gamble? Endocrine. In press. (doi:10.1007/s12020-015-0727-2)

4 Donohue A 2010 First Manic Episode in a 55-Year-Old Man After Initiation of Aripiprazole. Psychiatry 7 37-39. 
5 Peuskens J, Pani L, Detraux J \& De Hert M 2014 The effects of novel and newly approved antipsychotics on serum prolactin levels: a comprehensive review. CNS Drugs 28 421-453. (doi:10.1007/ s40263-014-0157-3)

6 Hoffer ZS, Roth RL \& Mathews M 2009 Evidence for the partial dopamine-receptor agonist aripiprazole as a first-line treatment of psychosis in patients with iatrogenic or tumorigenic hyperprolactinemia. Psychosomatics 50 317-324. (doi:10.1176/appi.psy.50.4.317)

7 Yasui-Furukori N, Furukori H, Sugawara N, Fujii A \& Kaneko S 2010 Dose-dependent effects of adjunctive treatment with aripiprazole on hyperprolactinemia induced by risperidone in female patients with schizophrenia. Journal of Clinical Psychopharmacology 30 596-599. (doi:10.1097/JCP.0b013e3181ee832d)

8 Li X, Tang Y \& Wang C 2013 Adjunctive aripiprazole versus placebo for antipsychotic-induced hyperprolactinemia: meta-analysis of randomized controlled trials. PLoS ONE 8 e70179. (doi:10.1371/ journal.pone.0070179)

9 Chen JX, Su YA, Bian QT, Wei LH, Zhang RZ, Liu YH, Correll C, Soares JC, Yang FD, Wang SL et al. 2015 Adjunctive aripiprazole in the treatment of risperidone-induced hyperprolactinemia: a randomized, double-blind, placebo-controlled, dose-response study. Psychoneuroendocrinology 58 130-140. (doi:10.1016/j.psyneuen.2015. 04.011)

10 Lozano R, Marin R \& Santacruz MJ 2014 Prolactin deficiency by aripiprazole. Journal of Clinical Psychopharmacology 34 539-540. (doi:10.1097/JCP.0000000000000151)

11 Saraf G, Behere RV, Venkatasubramanian G, Rao NP, Varambally S \& Gangadhar BN 2014 Hyperprolactinemia with aripiprazole: understanding the paradox. American Journal of Therapeutics 21 e80-e81. (doi:10.1097/MJT.0b013e3182456de7)

12 Chen S \& Hsiao Y 2010 Treatment with aripiprazole for hyperprolactinemia induced by pituitary microadenoma in a bipolar I disorder patient. Journal of Clinical Psychopharmacology 30 78-80. (doi:10.1097/ JCP.0b013e3181ca3c85)

13 Takeuchi H \& Remington G 2013 A systematic review of reported cases involving psychotic symptoms worsened by aripiprazole in schizophrenia or schizoaffective disorder. Psychopharmacology 228 175-185. (doi:10.1007/s00213-013-3154-1)

Received in final form 26 September 2015 Accepted 6 October 2015 